\title{
Effective Rigidity of a Polar Neutron Monitor for Recording Ground-Level Enhancements
}

\author{
Sergey A. Koldobskiy ${ }^{1,2}$. \\ Gennady A. Kovaltsov ${ }^{3}$. \\ Ilya G. Usoskin ${ }^{1,4}$
}

(C) Springer

\begin{abstract}
The "effective" rigidity of a neutron monitor for a GLE (groundlevel enhancement) event is defined so that the event-integrated fluence of solar energetic protons with rigidity above it is directly proportional to the integral intensity of the GLE as recorded by a polar neutron monitor, within a wide range of solar energetic-proton spectra. This provides a direct way to assess the integral fluence of a GLE event based solely on neutron monitor data. The effective rigidity/energy was found to be $1.13-1.42 \mathrm{GV}(550-800 \mathrm{MeV})$. A small model-dependent systematic uncertainty in the value of the effective rigidity is caused by uncertainties in the low-energy range of the neutron-monitor yield function, which requires more detailed computations of the latter.
\end{abstract}

Keywords: Cosmic rays, solar

\section{Introduction}

Even in the era of direct measurements of cosmic rays by space-borne instruments, including such sophisticated dedicated missions as Alpha Magnetic Spectrometer (AMS, Aguilar et al., 2015) and Payload for Antimatter Matter Exploration and Light-nuclei Astrophysics (PAMELA, Adriani et al., 2014), groundbased instruments remain the most important tool to monitor cosmic-ray variability. The most useful is the world-wide network of neutron monitors (NMs)

\footnotetext{
$\triangle$ I.G.Usoskin

ilya.usoskin@oulu.fi

1 Space Climate Research Unit, University of Oulu, Finland

2 National Research Nuclear University MEPhI, Moscow, Russia

3 Ioffe Physical-Technical Institute, St. Petersburg, Russia

4 Sodankylä Geophysical Observatory, University of Oulu, Finland
} 
which routinely measures the secondary nucleonic component of a cascade induced by energetic cosmic-ray particles in the Earth's atmosphere. The NM data recorded all over the world are collected in NMDB database (nmdb.eu) and are available nearly instantly online (Mavromichalaki et al., 2011). However, the NM is an energy-integrated device that cannot directly measure the energy spectrum of cosmic-ray particles (Shea and Smart, 2000; Simpson, 2000). For a fast analysis of the cosmic-ray variability as recorded by such an integral instrument, it is useful to know its characteristic energy. Sometimes a fixed energy, e.g. $10 \mathrm{GeV}$ (Belov, 2000), or the so-called median energy (Ahluwalia and Fikani, 2007), which halves the differential response function of NM to galactic cosmic rays (GCR), are considered as the characteristic energy of a NM. However, these approaches are very inaccurate, since the median energy varies over the solar cycle and thus cannot be a parameter of the detector itself. As an alternative, the concept of the "effective" energy of a NM to detect GCR has been introduced by Alanko et al. (2003) and further developed by Asvestari et al. (2017b). It is defined as the energy at which the variability of GCR is proportional to the recorded NM count rates. The effective energy of a polar NM to detect GCR was found to be $10-12 \mathrm{GeV}$.

In contrast to the permanently existing flux of GCR, modulated by solar activity during the solar magnetic cycle, there are sporadic solar eruptive events, when a massive bulk of solar energetic particles (SEPs) can bombard the Earth. Sometimes, the energy of SEPs can be sufficiently high to initiate an atmospheric cascade, and such events can be measured on the ground by NMs, leading to a special class of ground-level enhancement (GLE) events (Shea and Smart, 2012). The energy spectrum of SEP particles is much softer than that of GCR, and it is apparent that the effective energy defined for GCR is not applicable for the GLE events. It is not even obvious that such an effective energy should exist for the GLE detection by NMs. Here we estimate, for the first time, the effective energy of a standard sea-level polar NM to a GLE and discuss its applicability to the real data.

\section{The Concept of "Effective" Rigidity}

The spectrum of solar protons in the energy range typical for a GLE (i.e, as recorded by $\mathrm{NMs}$ ) is often described by a power law over the proton's rigidity (Shea and Smart, 2012). Accordingly, in this study we first considered the integral GLE omnidirectional proton fluence $F\left[\mathrm{~cm}^{-2}\right]$ in the following form

$$
F(>R)=F_{0} R^{-\gamma}
$$

where $\gamma$ is the spectral index, $R$ is the proton's rigidity, defined as

$$
R=\sqrt{E\left(E+2 E_{\mathrm{r}}\right)}
$$

$E$ is the proton's kinetic energy, $E_{\mathrm{r}}=0.938 \mathrm{GeV}$ is the proton rest mass. The spectral index $\gamma$ of the integral fluence takes values between 4 and 8 for GLE 
events (Raukunen et al., 2018). The (integrated over the entire event) response of a NM to a GLE can be presented, assuming the isotropic flux of SEPs, as

$$
N=\int_{R_{\mathrm{c}}}^{\infty} D(R) \mathrm{d} R
$$

where $R_{\mathrm{c}}$ is the local geomagnetic rigidity cutoff (e.g. Cooke et al., 1991), and $D(R)$ is the differential response function (Dorman, 2004):

$$
D(R)=Y_{\mathrm{p}}(R) \frac{\mathrm{d} F(>R)}{\mathrm{d} R},
$$

where $d F / d R$ is the differential spectrum of energetic particles, and $Y_{\mathrm{p}}$ is the so-called NM yield function for protons (e.g. Clem and Dorman, 2000). The yield function $\left[Y_{\mathrm{p}}(R)\right]$ is defined as the response of a standard NM to the unity fluence of protons with rigidity $R$. Here we use two yield functions recently provided by Mishev, Usoskin, and Kovaltsov (2013) and by Mangeard et al. (2016a), called Mi13 and Ma16, respectively, henceforth. Both were obtained as a result of full Monte-Carlo simulations of the nucleonic atmospheric cascade caused by primary energetic neutrons. They are presently the most accurate theoretical NM yield functions (Gil et al., 2015; Mangeard et al., 2016b), but have some slight differences between each other, especially in the low-energy range. These yield functions are shown in Figure 1 for a standard sea-level NM64 neutron monitor.

The differential response functions are shown in Figure 2 for the power-law spectrum of SEP (Equation 1) and the sea-level NM yield function according to Mi13. The curves are normalized to the unity total response of a polar NM (the area under the curves). The median rigidity, which halves the NM response, is denoted by the black dots. Sometimes (e.g. Bieber et al., 2013; Moraal and Caballero-Lopez, 2014), the median rigidity/energy is used to characterize the sensitivity of NM to SEP, but as one can see, it strongly depends on the spectral shape of SEPs and thus cannot be a characteristic of the detector.

Here we search for the so-called effective rigidity/energy of a NM for GLE protons. The effective rigidity $\left[R_{\text {eff }}\right]$ is defined so that the integral NM response to GLE protons $[N]$ is directly proportional to the total GLE fluence of protons with rigidity above $R_{\text {eff }}$ irrespective of the exact spectrum of protons:

$$
F\left(>R_{\text {eff }}\right)=K_{\text {eff }} N,
$$

where $K_{\mathrm{eff}}$ is (nearly) constant in the entire range of realistic GLE proton spectra.

Let us consider the coefficient

$$
K(R, \gamma)=F(>R) / N,
$$

which is the ratio of the proton integral fluence $[F]$ given by Equation 1 and the NM response $[N]$ calculated using Equation 3. Figure 3a depicts the dependence of $K$ on the rigidity $[R]$ computed for a polar sea level NM by applying the Mi13 yield function. One can see that indeed there is a specific value of the 


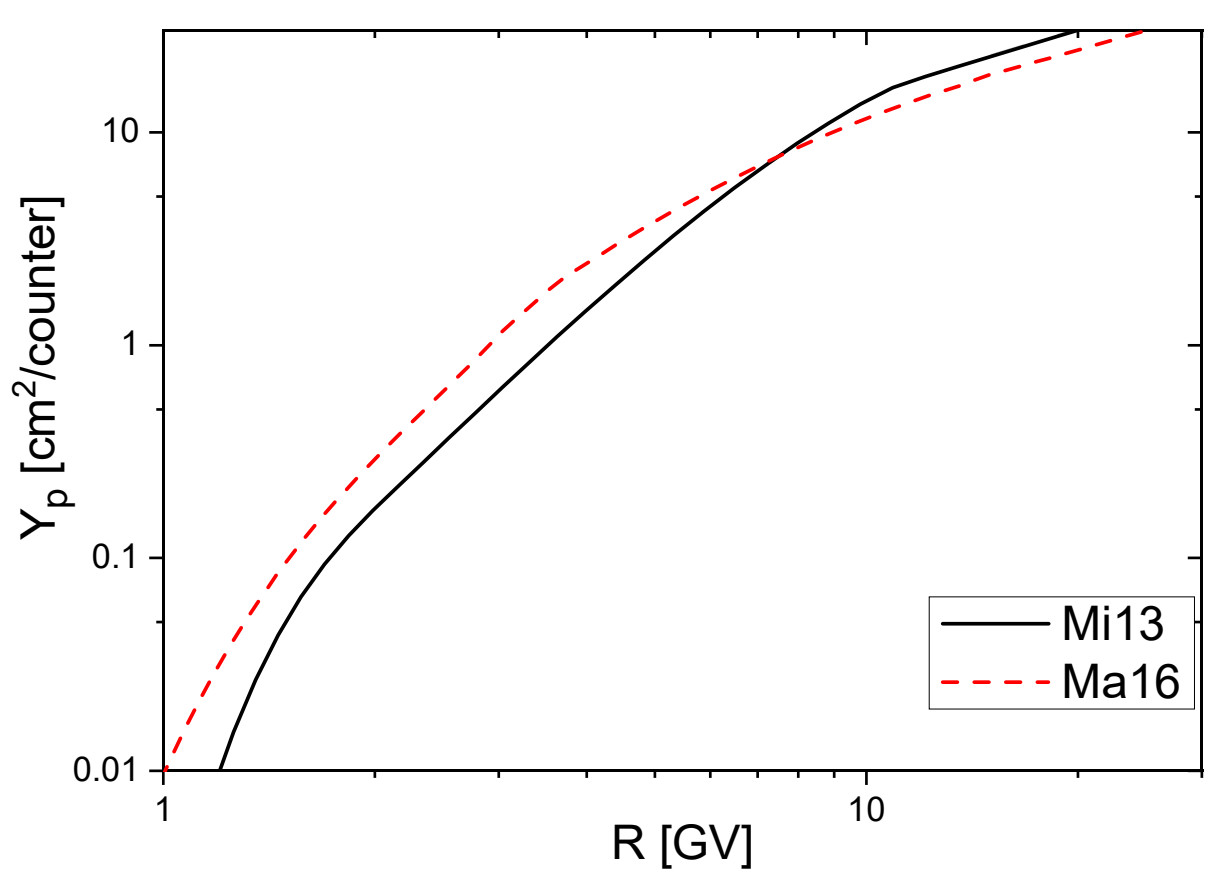

Figure 1. Low-energy part of neutron monitor yield functions $\left[\mathrm{cm}^{2}\right.$ per counter] as a function of the particle's rigidity $[R]$ according to Mishev, Usoskin, and Kovaltsov (2013, denoted as Mi13) and Mangeard et al. (2016a, Ma16)

proton rigidity, where all the curves intercept implying that the value of $K$ does not depend on the spectral index $[\gamma]$ for this rigidity. This value of rigidity corresponds to the effective rigidity $\left[R_{\mathrm{eff}}\right]$.

In order to quantify the search for the effective rigidity, we consider the quantity $\Delta K /\langle K\rangle$ as the merit function to be minimized. For a given value of $R, \Delta K$ is defined as the standard devisation of the $K$-values (the vertical range of different curves shown in Figure 3), and $\langle K\rangle$ is the mean $K$-value. The dependence of $\Delta K /\langle K\rangle$ on $R$ is shown in Figure 4 and depicts a clear minimum, which corresponds to the effective rigidity $R_{\text {eff }}$. The $K$-values at the effective rigidity remain constant, within the $10 \%( \pm 5 \%)$ interval, on the spectral index. This minimum $\Delta K /\langle K\rangle$ serves as the uncertainty of the $K$-value. The $R_{\text {eff }}$ value was found to be $1.46 \mathrm{GV}$ (or approximately $800 \mathrm{MeV}$ of the proton's kinetic energy) for the Mi13 yield function. For the Ma16 yield function, the value was $R_{\mathrm{eff}}=1.13 \mathrm{GV}$ (540 MeV of kinetic energy). The parameters (effective rigidity and energy, the proportionality coefficient $K_{\text {eff }}$, and its uncertainty) are listed in Table 1.

The calculations performed above validate the concept of "effective" rigidity, for which the the integral fluence of an SEP/GLE event can be simply estimated from the NM count rate. The above consideration was, however, based on an oversimplified assumption of the purely power-law shape of the SEP spectrum (Equation 1), which is known to be unrealistic. In the next section we study the 


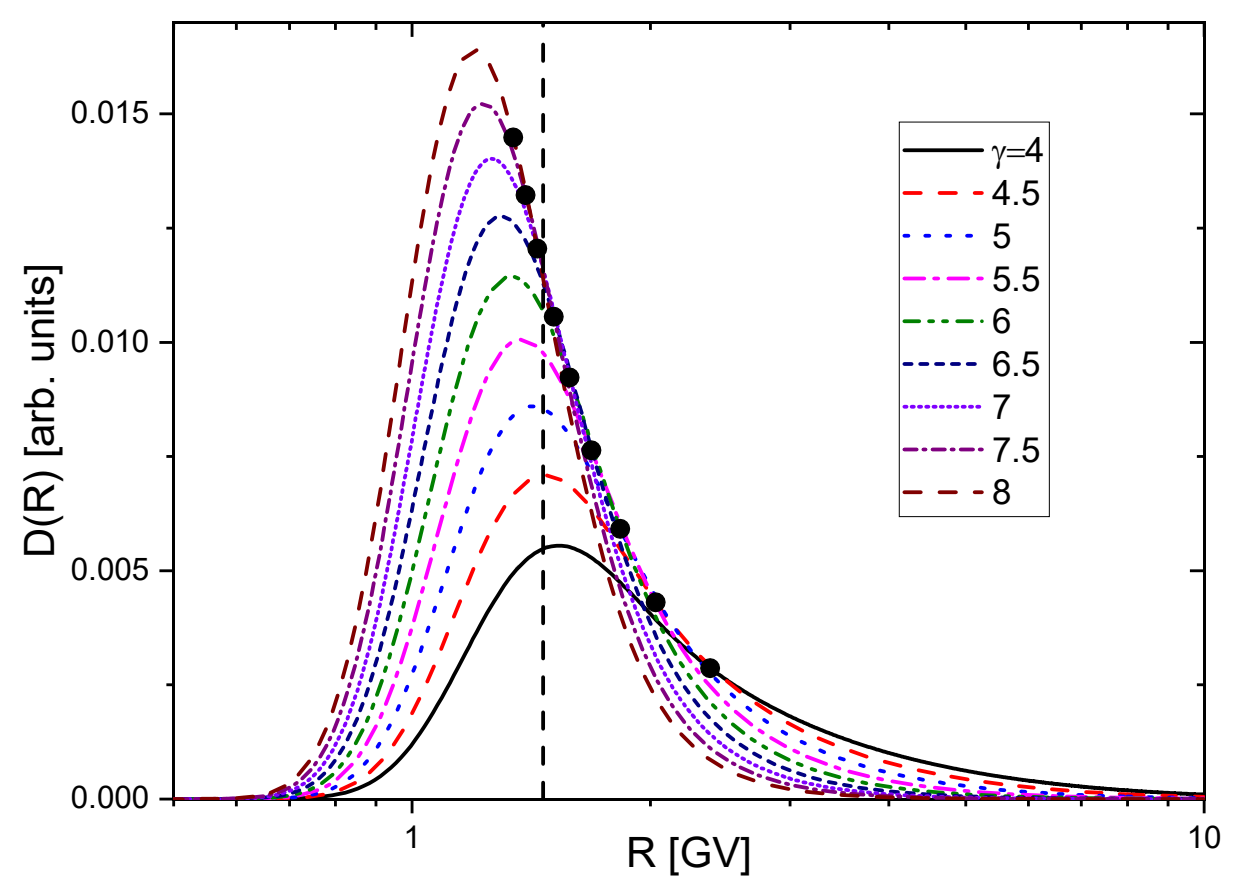

Figure 2. Normalized differential response functions of a sea-level NM for the power-law spectrum of SEP (Equation 1) and the Mi13 yield function. Different curves correspond to different values of the spectral index $\gamma$ as denoted in the legend. Black points denote the median rigidities for the shown functions, while the dotted line indicates the effective rigidity calculated here.
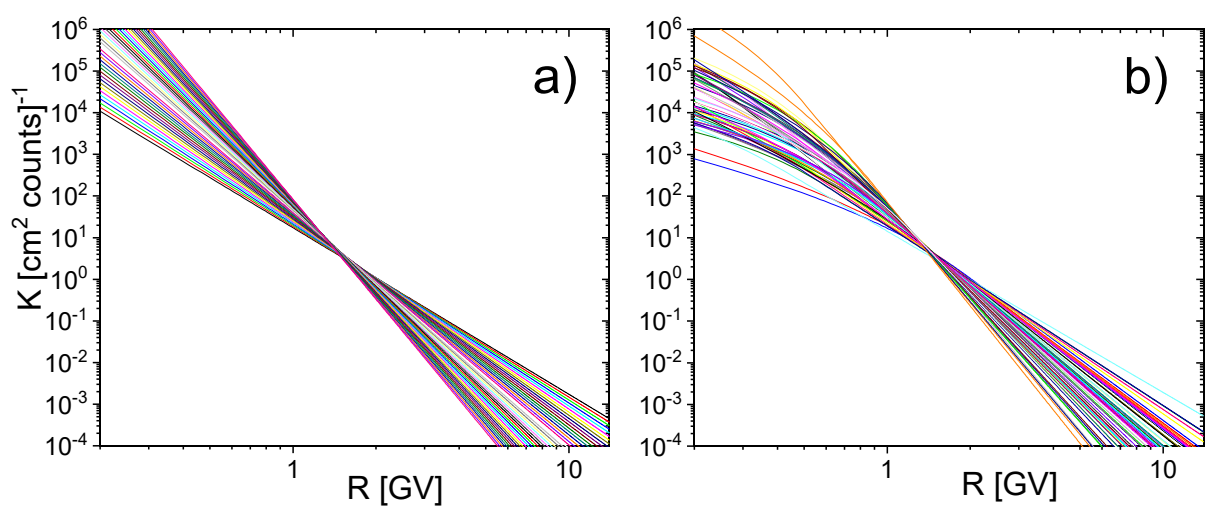

Figure 3. (a) Relationship between $K$ [per 1NM64] and the rigidity $R$ for power-law spectra with the spectral indices $[\gamma]$ ranging between 4 and 8 . Computations were performed using the Mi13 yield function. (b) The same as in panel a but for the realistic GLE spectra according to Raukunen et al. (2018). 

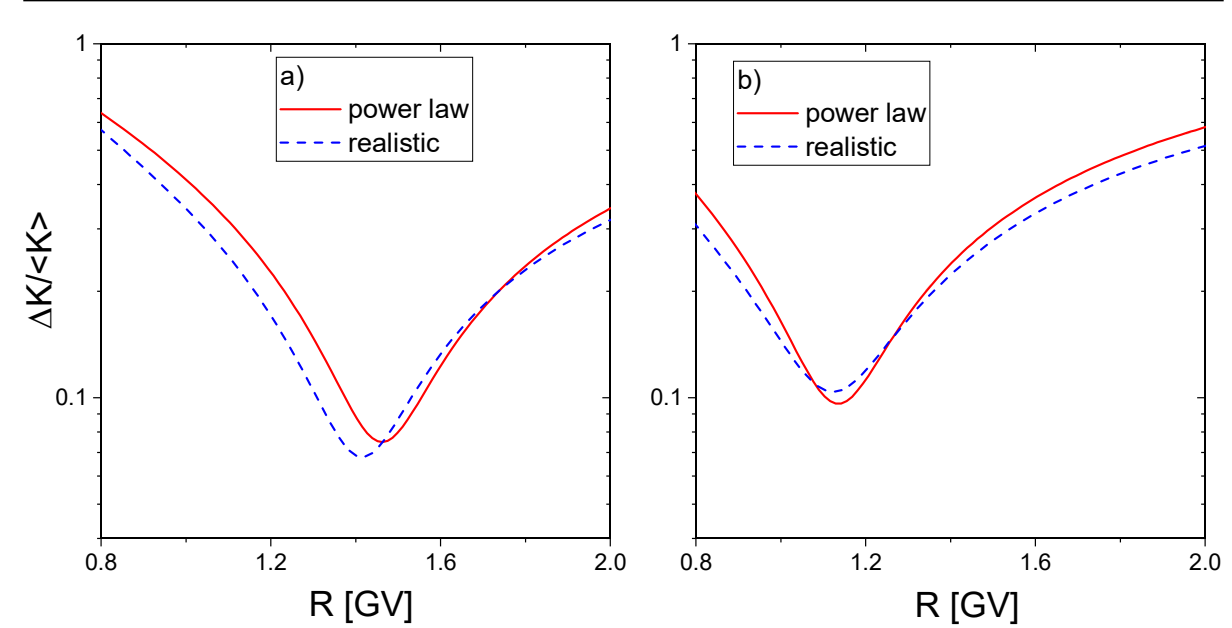

Figure 4. Dependence of $\Delta K /\langle K\rangle$ on the rigidity $[R]$. The red curves correspond to a simple power-law spectrum (Figure 3a), while dashed blue curves correspond to the realistic GLE spectra (Figure 3b). Panels a and b correspond to the NM yield functions according to Mishev, Usoskin, and Kovaltsov (2013) and Mangeard et al. (2016a), respectively.

Table 1. Values of the effective rigidity $\left[R_{\text {eff }}\right]$ energy, energy $\left[E_{\text {eff }}\right]$, the proportionality coefficient $\left[K_{\text {eff }}\right]$ per a standard 1NM64 counter, and its relative uncertainty $[\Delta K / K]$ for two spectrum models, power-law and realistic GLE, and two NM yield functions, Mi13 (Mishev, Usoskin, and Kovaltsov, 2013) and Ma16 (Mangeard et al., 2016a), for a polar neutron monitor.

\begin{tabular}{ccccc}
\hline & \multicolumn{2}{c}{ Mi13 } & \multicolumn{2}{c}{ Ma16 } \\
Parameters & Power law & Realistic & Power law & Realistic \\
\hline$R_{\text {eff }}[\mathrm{GV}]$ & 1.46 & 1.42 & 1.14 & 1.13 \\
$E_{\text {eff }}[\mathrm{GeV}]$ & 0.80 & 0.76 & 0.54 & 0.53 \\
$K_{\text {eff }}\left[\mathrm{cm}^{2} \text { counts }\right]^{-1}$ & 4.56 & 4.95 & 6.63 & 6.2 \\
$\Delta K / K$ & 0.076 & 0.068 & 0.097 & 0.104 \\
\hline
\end{tabular}

concept of effective rigidity using more realistic spectra of GLE events for the last decades.

\section{Application to Realistic GLE Spectra}

Strong SEP events with hard spectra can be detected by ground-based NMs and are called GLEs. Presently, there are registered 72 GLE events (see an official list at the International GLE Database gle.oulu.fi), of which integral spectra were reconstructed for 59 events (Tylka and Dietrich, 2009; Raukunen et al., 2018) using ground-based and space-borne measurements. Rigidity integral fluences (spectrum over the entire event) were reconstructed in the form of a Band function (Band et al., 1993), where the omnidirectional event-integrated fluence $F$ of 
an SEP event (in units of particles $\mathrm{cm}^{-2}$ ) can be represented as a broken powerlaw shape (softer at higher and harder at lower rigidities) with an exponential junction in between:

$$
F(>R)= \begin{cases}J_{0}\left(\frac{R}{1 \mathrm{GV}}\right)^{-\gamma_{1}} \exp \left(-\frac{R}{R_{0}}\right), & \text { if } R<R_{1} \\ J_{0}\left(\frac{R_{1}}{1 \mathrm{GV}}\right)^{-\gamma_{1}} \exp \left(-\frac{R_{1}}{R_{0}}\right)\left(\frac{R}{R_{1}}\right)^{-\gamma_{2}}, & \text { if } R \geqslant R_{1},\end{cases}
$$

where $J_{0}$ is an overall fluence normalization coefficient, $\gamma_{1}$ and $\gamma_{2}$ are the two power-law indices, $R_{0}$ is the characteristic rigidity, and $R_{1}=\left(\gamma_{2}-\gamma_{1}\right) R_{0}$ is the breakpoint rigidity. The coefficients $J_{0}, \gamma_{1}, \gamma_{1}$ and $R_{0}$ were taken from Raukunen et al. (2018), where they were obtained as a result of the Band-function fit to available ground-based and space-borne data. The validity of the Band-function parameterization of a GLE integral fluence has been verified by Asvestari et al. (2017a) using data from space-borne PAMELA data for the GLE No. 71 of 17 May 2012.

We repeated the analysis of the effective rigidity, as described in Section 2, but now applying realistic spectra corresponding to measured GLE events rather than an oversimplified power-law shape (Equation 1). The relationship between the proportionality coefficient $K$ and the particle rigidity is shown in Figure $3 \mathrm{~b}$ for the 59 analyzed spectra. One can see that, while the shape of the relations is not a power law, there is a clear indication of the existence of an effective rigidity at about $1.5 \mathrm{GV}$, where all the curves cross. The determination of the effective rigidity is shown in Figure 4 with blue dashed curves. One can see that values of both $R_{\text {eff }}$ and $\Delta K / K$ appear close to those for the pure power-low case, suggesting that the effective rigidity is defined quite robustly against the exact spectral shape. The corresponding values are listed in Table 1.

In order to illustrate how the concept of effective rigidity works, we show in Figure 5 the dependence of the proportionality coefficient $K$ on $\gamma_{2}$ (the highrigidity index of the Band-function approximation) for different values of rigidity $R$. The dependence is nearly flat, within the range of $7 \%$ for the effective rigidity of $1.46 \mathrm{GV}$. However, even a slight change of the rigidity distorts the dependence significantly and systematically.

As a final test for the effective rigidity concept, we show in Figure 6 a relation between the integral fluence above the effective rigidity $F\left(>R_{\text {eff }}\right)$ and the measured integral intensity (Asvestari et al., 2017a) for all of the analyzed GLE events. The black and red dots correspond to the values of $R_{\text {eff }}=1.46 \mathrm{GV}$ and $1.14 \mathrm{GV}$ as defined for the Mi13 and Ma16 yield functions, respectively. One can see that both dependencies are quite well linear but slightly offset because of the different values of $R_{\text {eff }}$. The squared Pearson's correlation coefficient $r^{2}$ is 0.96 and 0.94 , for the Mi13 and Ma16 yield functions, respectively, implying that the former one provides a non-significantly better agreement. While the overall agreement is good, some points lie essentially off the main dependence. This is likely related to GLE events with two distinguishable phases observed: a first prompt GLE phase followed by a delayed component, the so-called energetic storm particle phase or ESP (Raukunen et al., 2018). We note that the majority of the analyzed events have only the GLE main phase. For such two-phase events 


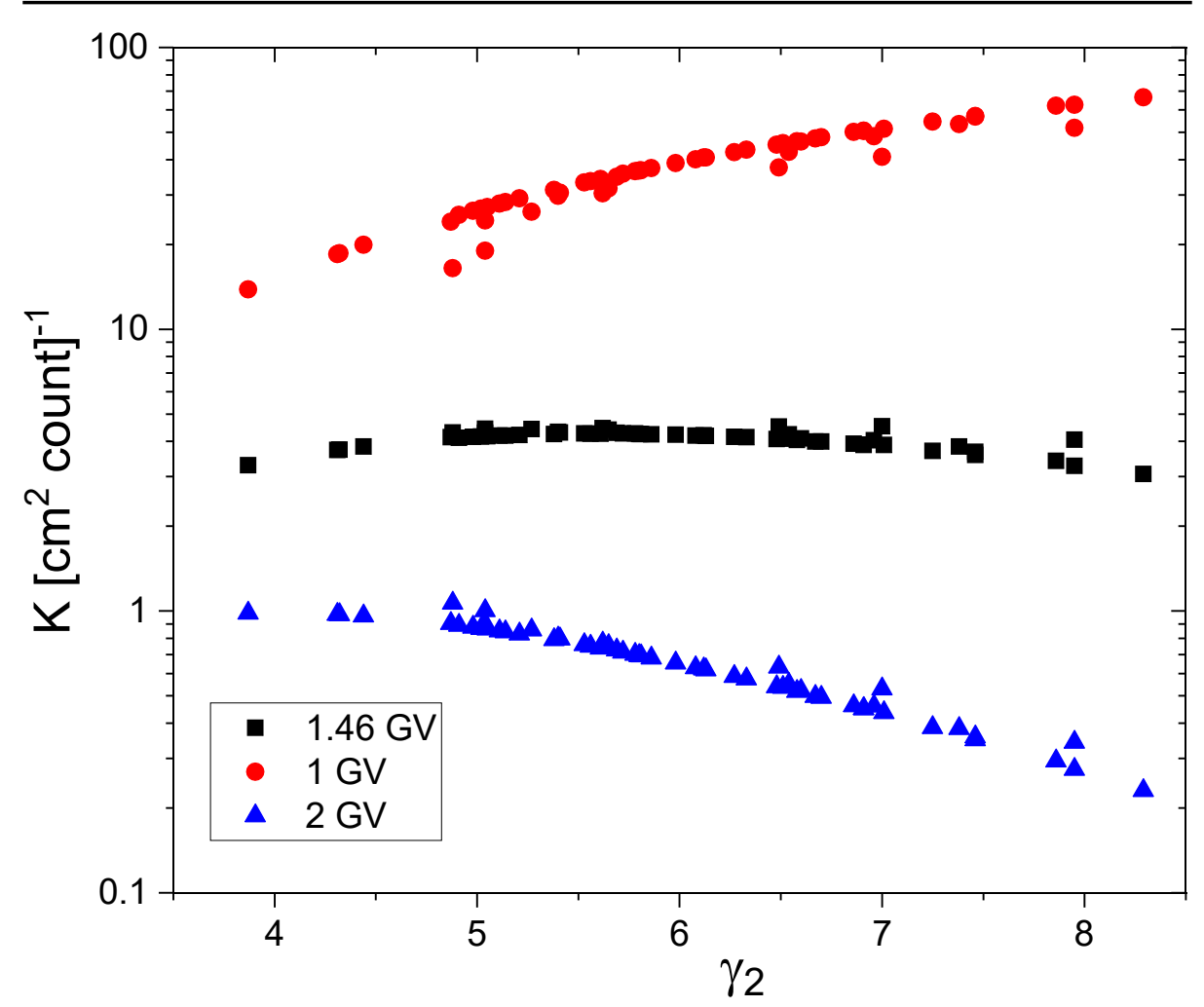

Figure 5. Relationship between $K$-coefficients (per 1NM64) and the Band function high rigidity power-law index $\gamma_{2}$ for 59 analyzed GLE events, using three different values of the rigidity, as denoted in the legend ( $1.46 \mathrm{GV}$ corresponds to $\left.R_{\text {eff }}\right)$ and applying the Mi13 yield function.

(GLE Nos. 43, 59, 62, 65) only the first GLE phase was taken into account. As a result, the integral fluence may be somewhat underestimated for these events. For GLE 42, with a very anisotropic first phase, the ESP phase was also considered for computations of the integral fluence.

\section{Discussion and Conclusions}

We have shown that there exists an effective rigidity $\left[R_{\mathrm{eff}}\right]$ of a neutron monitor that is defined so that the event-integrated fluence of solar energetic protons with rigidity above it, $F\left(>R_{\text {eff }}\right)$, for a GLE event is directly proportional to the integral intensity of the GLE as recorded by a polar neutron monitor (Equation 5). This definition is robust, and the direct relation works within a wide range of the solar energetic proton spectra, including both simple theoretical models and realistic estimates for historical GLE events. This provides a direct way to perform a fast and accurate assessment of the integral fluence of a GLE event based solely on the recorded enhancement of a NM. The exact value of the effective rigidity was found to be, for a polar neutron monitor, between 


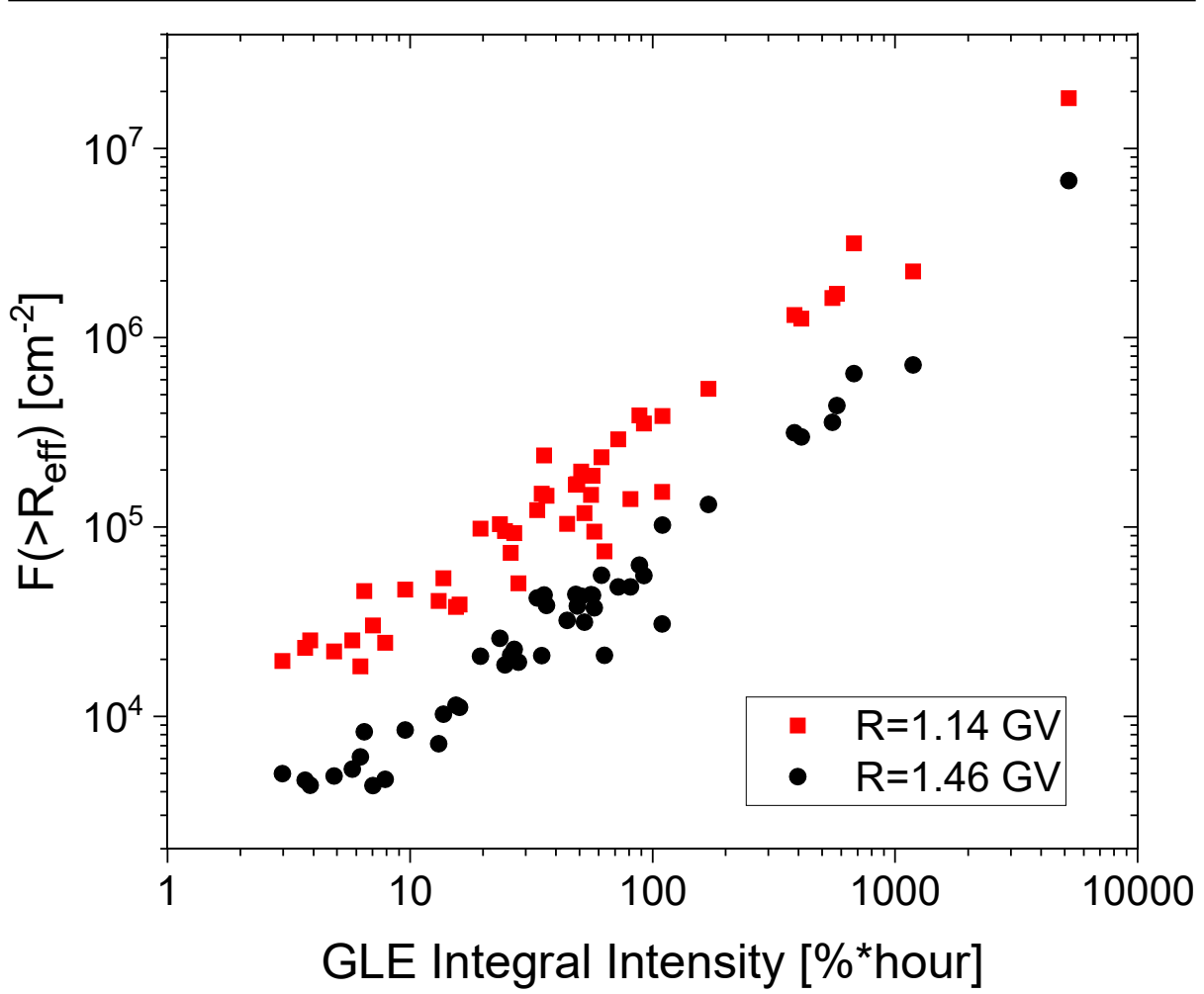

Figure 6. Relationship between the integral fluence $\left[F\left(>R_{\text {eff }}\right)\right]$ and the integral intensity recorded by polar neutron monitors for the 59 GLEs analyzed here. The black and red dots correspond to the $R_{\text {eff }}$ obtained for the Mi13 and Ma16 yield functions, respectively.

$1.13-1.43 \mathrm{GV}$ ( $540-760 \mathrm{MeV}$ of proton's kinetic energy) and the $K$-coefficient $4.95-6.2\left(\mathrm{~cm}^{2} \text { count }\right)^{-1}$ for realistic spectra and polar NM (see Table 1). The found effective rigidity/energy is significantly higher than that of cosmogenic isotope production for GLE events $(\approx 200 \mathrm{MeV}$, see Kovaltsov et al., 2014). This is because cosmogenic isotopes $\left({ }^{10} \mathrm{Be}\right.$ and $\left.{ }^{14} \mathrm{C}\right)$ are mostly produced in the stratosphere, while development of a full cascade reaching the ground, and thus higher rigidity/energy of primary particles, is required to cause a GLE event.

We note that the the result (exact values of $R_{\text {eff }}$ and $K_{\text {eff }}$ ) is slightly modeldependent as caused by a difference between the used NM yield-functions in low-energy range (Figure 1), which is known but whose origin is unclear. This discrepancy introduce a slight (within $\pm 10 \%$ ) systematic uncertainty in the result, and more detailed studies of the NM yield function in the low-energy range are necessary to resolve this discrepancy.

Acknowledgments This work was partially supported by the ReSoLVE Centre of Excellence (Academy of Finland, project 272157). S.A. Koldobsky acknowledges the President's of the Russian Federation grant no. MK-6160.2018.2. 


\section{Disclosure of Potential Conflicts of Interest}

The authors declare that they have no conflicts of interest.

\section{References}

Adriani, O., Barbarino, G.C., Bazilevskaya, G.A., Bellotti, R., Boezio, M., Bogomolov, E.A., Bongi, M., Bonvicini, V., Bottai, S., Bruno, A., et al.: 2014, The PAMELA Mission: Heralding a new era in precision cosmic ray physics. Phys. Rep. 544, 323. DOI. ADS.

Aguilar, M., Aisa, D., Alpat, B., Alvino, A., Ambrosi, G., Andeen, K., Arruda, L., Attig, N., Azzarello, P., Bachlechner, A., et al.: 2015, Precision Measurement of the Proton Flux in Primary Cosmic Rays from Rigidity $1 \mathrm{GV}$ to $1.8 \mathrm{TV}$ with the Alpha Magnetic Spectrometer on the International Space Station. Phys. Rev. Lett. 114(17), 171103. DOI. ADS.

Ahluwalia, H.S., Fikani, M.M.: 2007, Cosmic ray detector response to transient solar modulation: Forbush decreases. J. Geophys. Res. 112, A08105. DOI. ADS.

Alanko, K., Usoskin, I.G., Mursula, K., Kovaltsov, G.A.: 2003, Heliospheric modulation strength: Effective neutron monitor energy. Adv. Space Res. 32, 615. DOI.

Asvestari, A., Willamo, T., Gil, A., Usoskin, I.G., Kovaltsov, G.A., Mikhailov, V.V., Mayorov, A.: 2017a, Analysis of Ground Level Enhancements (GLE): Extreme solar energetic particle events have hard spectra. Adv. Space Res. 60, 781. DOI.

Asvestari, E., Gil, A., Kovaltsov, G.A., Usoskin, I.G.: 2017b, Neutron Monitors and Cosmogenic Isotopes as Cosmic Ray Energy-Integration Detectors: Effective Yield Functions, Effective Energy, and Its Dependence on the Local Interstellar Spectrum. J. Geophys. Res. (Space Phys.) 122, 9790. DOI. ADS.

Band, D., Matteson, J., Ford, L., Schaefer, B., Palmer, D., Teegarden, B., Cline, T., Briggs, M., Paciesas, W., Pendleton, G., Fishman, G., Kouveliotou, C., Meegan, C., Wilson, R., Lestrade, P.: 1993, BATSE observations of gamma-ray burst spectra. I - Spectral diversity. Astrophys. J. 413, 281. DOI. ADS.

Belov, A.: 2000, Large Scale Modulation: View From the Earth. Space Sci. Rev. 93, 79. DOI. ADS.

Bieber, J.W., Clem, J., Evenson, P., Pyle, R., Sáiz, A., Ruffolo, D.: 2013, Giant Ground Level Enhancement of Relativistic Solar Protons on 2005 January 20. I. Spaceship Earth Observations. Astrophys. J. 771, 92. DOI. ADS.

Clem, J.M., Dorman, L.I.: 2000, Neutron monitor response functions. Space Sci. Rev. 93, 335. DOI. ADS.

Cooke, D.J., Humble, J.E., Shea, M.A., Smart, D.F., Lund, N., Rasmussen, I.L., Byrnak, B., Goret, P., Petrou, N.: 1991, On cosmic-ray cut-off terminology. Nuovo Cimento C 14, 213. ADS.

Dorman, L.I.: 2004, Cosmic rays in the earth's atmosphere and underground, Kluwer, Dordrecht.

Gil, A., Usoskin, I.G., Kovaltsov, G.A., Mishev, A.L., Corti, C., Bindi, V.: 2015, Can we properly model the neutron monitor count rate? J. Geophys. Res. 120, 7172. DOI. ADS.

Kovaltsov, G.A., Usoskin, I.G., Cliver, E.W., Dietrich, W.F., Tylka, A.J.: 2014, Fluence Ordering of Solar Energetic Proton Events Using Cosmogenic Radionuclide Data. Solar Phys. 289, 4691. DOI. ADS.

Mangeard, P.-S., Ruffolo, D., Sáiz, A., Nuntiyakul, W., Bieber, J.W., Clem, J., Evenson, P., Pyle, R., Duldig, M.L., Humble, J.E.: 2016a, Dependence of the neutron monitor count rate and time delay distribution on the rigidity spectrum of primary cosmic rays. J. Geophys. Res. (Space Phys.) 121, 11620. DOI. ADS.

Mangeard, P.-S., Ruffolo, D., Sáiz, A., Madlee, S., Nutaro, T.: 2016b, Monte Carlo simulation of the neutron monitor yield function. J. Geophys. Res. 121, 7435. DOI. ADS.

Mavromichalaki, H., Papaioannou, A., Plainaki, C., Sarlanis, C., Souvatzoglou, G., Gerontidou, M., Papailiou, M., Eroshenko, E., Belov, A., Yanke, V., Flückiger, E.O., Bütikofer, R., Parisi, M., Storini, M., Klein, K.-L., Fuller, N., Steigies, C.T., Rother, O.M., Heber, B., WimmerSchweingruber, R.F., Kudela, K., Strharsky, I., Langer, R., Usoskin, I., Ibragimov, A., Chilingaryan, A., Hovsepyan, G., Reymers, A., Yeghikyan, A., Kryakunova, O., Dryn, E., Nikolayevskiy, N., Dorman, L., Pustil'Nik, L.: 2011, Applications and usage of the real-time Neutron Monitor Database. Adv. Space Res. 47, 2210. DOI. ADS. 
Mishev, A.L., Usoskin, I.G., Kovaltsov, G.A.: 2013, Neutron monitor yield function: New improved computations. J. Geophys. Res. 118, 2783. DOI. ADS.

Moraal, H., Caballero-Lopez, R.A.: 2014, The Cosmic-Ray Ground-level Enhancement of 1989 September 29. Astrophys. J. 790, 154. DOI. ADS.

Raukunen, O., Vainio, R., Tylka, A.J., Dietrich, W.F., Jiggens, P., Heynderickx, D., Dierckxsens, M., Crosby, N., Ganse, U., Siipola, R.: 2018, Two solar proton fluence models based on ground level enhancement observations. J. Space Weather Space Clim. 8(27), A04. DOI. ADS.

Shea, M.A., Smart, D.F.: 2000, Fifty Years of Cosmic Radiation Data. Space Sci. Rev. 93, 229. DOI. ADS

Shea, M.A., Smart, D.F.: 2012, Space Weather and the Ground-Level Solar Proton Events of the 23rd Solar Cycle. Space Sci. Rev. 171, 161. DOI. ADS.

Simpson, J.A.: 2000, The Cosmic Ray Nucleonic Component: The Invention and Scientific Uses of the Neutron Monitor - (Keynote Lecture). Space Sci. Rev. 93, 11. DOI. ADS.

Tylka, A., Dietrich, W.: 2009, A new and comprehensive analysis of proton spectra in groundlevel encahnced (GLE) solar particle events. In: 31th Internat. Cosmic Ray Conf., icrc0273. Universal Academy Press, Lodź, Poland. 